\title{
Genetic Potential for Growth, Feed Conversion and Longevity
}

\author{
V. L. Stass ${ }^{1}$ \\ ${ }^{1}$ Laboratory of Animal Genetics, Institute of Animal Husbandry and Veterinary Sciences, Latvia \\ Correspondence: V. L. Stass, Laboratory of Animal Genetics, Institute of Animal Husbandry and Veterinary \\ Sciences, Latvia. E-mail: valbet.axon@apollo.lv
}

Received: November 15, 2018 Accepted: December 17, $2018 \quad$ Online Published: December 23, 2018

doi:10.5539/ijb.v11n1p66

URL: https://doi.org/10.5539/ijb.v11n1p66

\begin{abstract}
The purpose of this study was to find out genetic potential for growth, and feed conversion coefficient in pigs. It was done by analysing the relationship between variables that are relevant to animals' development, namely the growth rate, feed conversion coefficient, and live weight.

The study entails a hybrid dynamic mathematical model of the traits studied. The model is a species-specific concept, it was built for growing domestic pigs. Pigs are well-known model animals in human physiology. Some physiological factors are reportedly control both growth and ageing. Growth hormone and insulin-like growth factor-1 are reportedly the factors, which modulate growth, aging, and body size in mammals. The model does not entail growth hormone and insulin-like growth factor-1 as variables. However, the study demonstrates nonlinear dynamic of relevant variables in domestic pigs from $30 \mathrm{~kg}$ up to $600 \mathrm{~kg}$. The model was constructed by considering functional relations between variables analysed in experiments and field observations. Theoretical notions about growth processes as a dynamic system are included in the model. An invariant of growth dynamic is introduced. The study suggests that growth, feed conversion, and life span are functionally related traits in domestic pigs. In the pig, longevity is a function of growth rate and feed conversion coefficient.

The novelty of the study is a method for the investigation of genetic potential for growth, and longevity in pigs. In the model, genetic potential for growth rate and feed conversion were identified by analysing functional relations between relevant variables. The concept supports the opinion that growth, and ageing are interrelated processes.
\end{abstract}

Keywords: Animal Development, Ontogenetic Growth, Growth Invariant, Maximum Weight, Feed Conversion, Life Span, Hybrid Model

\section{Introduction}

\subsection{Genetic Potential}

The aim of this study was to find out the genetic potential for the growth, and feed conversion in pigs. Genetic potential was considered as maximum performance of a quantitative trait, a phenotype, that an animal is able to develop under optimal environmental conditions. In human physiology, pigs are well-known model animals (Walters et al., 2012). Experimental, empirical research into the problem is hardly tenable. The feasible way of approaching this task is an analytical method for analysing functional relations between relevant variables. The method applied to meet the study purpose was mathematical modelling. The concept is presented in the form of a hybrid model formulated as a dynamic system. The model is a combination of partial difference equation technique with discrete chronological time, and standard continuum methods.

\subsection{Life Span and Growth}

In this section, biological functions common for the traits studied have been discussed. Aging has been characterized by deterioration in the maintenance of homeostatic processes over time, leading to functional decline and increased risk for disease and death. Metabolically, insulin resistance, changes in body composition, physiological declines in growth hormone and insulin-like growth factor-1 characterize the aging process (Barzilai et al., 2012). A picture is emerging of a signalling network that is sensitive to nutritional status and that controls growth, stress resistance, and aging (Gems \& Partridge, 2013). Notably, growth and life span in animals are modulated by most of the abovementioned physiological factors. Specifically, growth hormone and 
insulin-like growth factor-1 are thought to modulate both growth and longevity thus suggesting a physiological base for a mathematical model of the biologically coupled traits.

Growth hormone and insulin-like growth factor-1 are reportedly the physiological factors, which influence or regulate growth, longevity, and aging in animals. Similarities among insulin-IGF-1-like signalling pathways in invertebrates and mammals reveal evidence for evolutionary conservation, raising the possibility that similar genetic modifications to the insulin-IGF-1 signalling cascade may extend life span in mammals (Carter et al., 2002). It is becoming clear that the insulin-signalling network is a key player that integrates not only metabolism and the response to nutrition, but also the regulation of cell death, ageing, and longevity, as well as the regulation of growth and body size (Nijhout, 2003).

Nutritional intake is another systemic factor that strongly modulates growth but does not appear to be the predominant mechanism driving normal growth deceleration. Although under nutrition inhibits juvenile growth, over nutrition does not prevent growth deceleration (Lui \& Baron, 2011).

Genetic determination of the traits is the next factor to consider. Life span and longevity are complex and multi-factorial traits resulting from a combination of unique reciprocal interaction between environmental, genetic, epigenetic, and stochastic factors, each contributing to the overall phenotype (Ostan et al., 2016). Several models have been identified through which genes are shown to play a major role in the extension of life span (Mitchell et al., 2015). Ontogenetic growth in animals is also under genetic control (Wu et al., 2003). Evidence suggests that the normal process of growth is driven, at a physiological level, by growth itself and, at a molecular level, by a genetic programme (Lui \& Baron, 2011). What is noticeable is that pathways controlling aging, including growth hormone and insulin-like growth factor-1, also control growth, therefore suggesting that growth causes aging (Gems \& Partridge, 2013), and implicitly controls longevity.

\subsection{Ontogenetic Growth}

Growth and development are complex nonlinear biological processes. In this study, one aspect of development, namely growth in animals was modelled. In spite of their obvious importance, the genetic, developmental, and physiological mechanisms that control age and size at maturity are for the most part unknown. Nutrition and hormones play obvious and well-established roles in growth, but the natural mechanisms that cause the cessation of growth when an animal reaches a species-specific size remain among the great puzzles in biology (Nijhout, 2015; Vollmer et al., 2017). Body growth in animals is rapid in early life but then progressively slows, thus imposing a limit on adult body size. Evidence suggests that this progressive decline in growth results from a genetic programme that occurs in multiple organs and involves the down-regulation of a large set of growth-promoting genes. This genetic programme does not appear to be driven simply by time, but rather depends on growth itself, suggesting that the limit on adult body size is imposed by a negative feedback loop (Lui \& Baron, 2011). This suggests that growth dynamic in animals is highly nonlinear (Suki \& Frey, 2017), phenotype-dependant, and physiologically complicated processes that entails regulatory interactions between traits (Ostan et al., 2016).

\subsection{Mathematical Model}

Many approaches have been proposed to model growth as a function of time such as exponential, sigmoid, or even more complex mathematical functions, most of which are descriptive in nature or describe population level phenomena (Suki \& Frey, 2017). Reportedly, growth functions currently used in industry serve the purpose well. However, they are inadequate tools for scientific explanation of the growth and longevity in animals. Although many of the models that have been advanced have significant attractions, none can be accepted without serious reservations, and the possibility that no one model can fit all cases has to be more seriously entertained (Agutter \& Wheatley, 2004). Applying Pütter equation or its derivatives such as the von Bertalanffy, the Gompertz, logistic and some others (Boukal et al., 2014), several quasi-explanatory models have been proposed for the allometric scaling of metabolic rate with body weight. Most of them have considerable appeal, particularly the recent ones, but none of them can be unconditionally accepted; no model has yet addressed every relevant issue (Agutter \& Wheatley, 2004). If the aim is to provide a phenomenological description of a growth trajectory and stop there, shortcomings of the Pütter model and its variants, such as the von Bertalanffy, the Gompertz, and logistic growth models do not apply. However, when a more mechanistic description of growth is needed, the limitations of these models become increasingly restrictive (Boukal et al., 2014). Research into association between the growth and longevity based on a population, heard or a breed analyses, or applying one of the abovementioned growth functions, can hardly be accepted while does not serve the study purpose. To provide rational analysis, an analytical, deterministic model of individual growth in animals was built. 
The coexistence of continuous and discrete dynamics characterizes hybrid dynamical systems. Modelling by hybrid dynamical systems is particularly important for understanding the nonlinear dynamics of biological and medical systems as they have many discontinuities. These systems can be mathematically described as hybrid dynamical systems with continuous and discrete variables (Aihara \& Suzuki, 2010). Such systems fall into broad, overlapping classes: impacting, piecewise smooth, and hybrid systems with a mixture of continuous and discrete time dynamics. They are often used to capture the behaviour of systems that contain some sort of discontinuous events (Di Bernardo \& Hogan, 2010). In concise form: wherever continuous and discrete dynamics interact, hybrid systems arise. These mathematical models come in all kinds of variations, but mostly, consist of some form of differential or difference equations (Heemels et al., 2010).

\section{Materials and Methods}

The data set was obtained from experiments on growing domestic pigs, LW, fed from $30 \pm 6 \mathrm{~kg}$ up to $96 \pm 4 \mathrm{~kg}$ live weight. The pigs were housed and fed under non-industrial conditions, either in a pig testing station or in research facilities. The animals were kept loose in groups of up to four to a pen, or individually in pens, fed a dry balanced feed with unlimited access to water contingent on the experiment design, ad libitum, or a constrained diet, in accordance with current body weight, adjusting feed quantity once a week. The investigation of animals' growth was carried out in compliance with Declaration of Helsinki, National legislation, and institutional rules.

\subsection{The Model's Variables}

' $M$ ' stands for an individual animal current live weight, measured in kilograms.

$M=\left\{\mathrm{M} \in \mathbb{R}_{+} \mid 30 \leq M \leq 600\right\}$, an animal individual maximum weight, $M_{\mathrm{x}}=600 \mathrm{~kg}$.

' $m$ ' is an animal initial considered weight, measured in kilograms, $M \geq m, m_{0}=30 \mathrm{~kg}$.

' $t$ ' is chronological discrete current time, measured in days starting from animal birth.

$t=\{t \in \mathbb{N} \mid 0<t<\infty\}, \Delta t=1,2,3 \ldots \mathrm{n}, \mathrm{n} \in \mathbb{N}$.

' $t_{0}$ ' is starting time, correspond with $m_{0}, t_{0}=90$ days.

' $K$ ' is an invariant of growth, considered as a parameter, nondimensional.

$K=\left\{K \in \mathbb{R}_{+} \mid 1 \leq K<11\right\}, K_{0}=1$.

$' Z$ is a current feed conversion coefficient, dimensionless.

$Z=\left\{Z \in \mathbb{R}_{+} \mid Z_{0} \leq Z<\infty\right\},(Z=\infty) \rightarrow\left(M=M_{\max }\right), Z_{\mathrm{o}}$ correspond with $m_{\mathrm{0}}$.

\subsection{Methodological Details}

There are two methodological aspects to mention. Almost all known organisms age, and the maximum life span differs between individual organisms (Mitchell et al., 2015). A problem with maximum life span is that it refers to a single event in a single individual animal. Because this is a stochastic event, the value for a single individual may be unrepresentative of the general trend. This raises another related point, that the maximum is itself dependent on the sample size of individuals included in the sample (Speakman, 2005).

This deficiency of statistical analyses is also attributable to the growth analyses of an individual animal. Namely, there is no clear association between the variability of individual growth and the variability of population growth, and thus population-based growth charts are not suitable to predict the variability of individual growth trajectories. Population based growth charts are not able to account for the large variability of individual growth patterns (Suki \& Frey, 2017). This suggests that in order more effectively select domestic crop and animal species for improved production traits, we need to go beyond these statistical descriptions and to overcome the very impediment that necessitated the biometrical approach to the analysis of quantitative traits (Mackay, 2001). It follows that analytical modelling of quantitative traits dynamic in an individual animal is the way to go to clarify causative relations between analysed quantitative traits.

\section{Results}

\subsection{Ontogenetic Growth}

Ontogenetic growth has been modelled by the same set of variables in all stages of development. Two main variables, the growth invariant $\mathrm{K}$, and the feed conversion coefficient, $\mathrm{Z}$ were considered.

The model does not have unknown speculative parameters, the parameters identification problem does not exist. It was possible due to the modelling approach. In the model, only functional relations between variables found in experiments and field observations were included. Speculative assumptions were not considered. 


\subsubsection{Rapid Growth}

Rapid growth in domestic pigs has been observed between $30 \mathrm{~kg}$ and $96 \mathrm{~kg}$ live weight. Parameter $\mathrm{K}$ is an invariant of growth, it is equal for the same weight pigs regardless of their daily gain. The growth invariant $\mathrm{K}$ has the following form:

$$
K=\frac{M t}{m_{o}\left(2 t-t_{o}\right)}
$$

Equation (1) can be written in a few different forms. The following two have usually been used.

$$
\begin{gathered}
\frac{M}{m_{o}}=2 K-1+\frac{t-K t_{o}}{t} \\
\frac{1}{m_{o}} \cdot \frac{\Delta M}{\Delta t}=\frac{\Delta K}{\Delta t}+\frac{K}{t}, \text { where } \Delta t=t-t_{o}, \Delta M=M-m_{o} .
\end{gathered}
$$

The relations between variables, in general form are formulated by the following system

$$
\left\{\begin{array}{l}
\frac{M}{m_{o}}=2 K-1+\frac{\left(t-K t_{o}\right)}{t} \\
\frac{\left(t-K t_{o}\right)}{t}=\frac{(Z-2 K) \cdot(K-1)}{Z K}
\end{array}\right.
$$

Equation (1) and system (4) are mathematical relations between the analysed variables found in the experiments. We start analyses of a rapid growth model. The rapid growth in domestic pigs has been observed in live weight range between $30 \mathrm{~kg}$ and $96 \mathrm{~kg}$. In this weight range, the growth in pigs has been modelled well by the following equation

$$
\frac{M}{m_{o}}=2 K-1
$$

The following two systems describe relations between variables during rapid growth, (6) and (7).

$$
\begin{gathered}
\left\{\begin{array}{l}
\frac{M}{m_{o}}=2 K-1 \\
\frac{\Delta M}{\Delta K}=\frac{2 M}{2 K-1}
\end{array}\right. \\
\left\{\begin{array}{l}
\frac{M}{m_{o}}=2 K-1+\frac{\left(t-K t_{o}\right)}{t} \\
\frac{\left(t-K t_{o}\right)}{t}=0 \\
\frac{\left(t-K t_{o}\right)}{t}=\frac{(Z-2 K) \cdot(K-1)}{Z K}
\end{array}\right.
\end{gathered}
$$

From (6) and (7) follow two equations that we consider first.

$$
\begin{gathered}
\frac{1}{m_{o}} \cdot \frac{\Delta M}{\Delta t}=\frac{1}{t} \cdot\left[\frac{2 K M}{2 K M-m_{o}(2 K-1)^{2}}\right], \quad m_{o} \leq M \leq 96 . \\
\frac{1}{m_{o}} \cdot \frac{\Delta M}{\Delta t}=\frac{1}{t} \cdot\left[\frac{2 K m_{o}(2 K-1)}{2 K M-m_{o}(2 K-1)^{2}}\right], \quad m_{o} \leq M \leq 96 .
\end{gathered}
$$

Equations (8) and (9) describe the rapid growth. Under condition

$$
2 K M-m_{o}(2 K-1)^{2}=0
$$

maximum growth rate is expected. Considering $M=96 \mathrm{~kg}$ in (9.1), it follows $\mathrm{K}=2.50$. As a result, the shortest time span needed for an animal to reach $96 \mathrm{~kg}$ is $\mathrm{t}_{(\mathrm{M}=96) \wedge(\mathrm{K}=2.50)}=125$ days. It means that maximum average growth rate during rapid growth in the weight range between $30 \mathrm{~kg}$ and $96 \mathrm{~kg}$ is $1.885 \mathrm{~kg}$ a day. This result follows from the calculation: $\Delta \mathrm{M}=66 \mathrm{~kg}$, and $\Delta \mathrm{t}=35$ days. It means that most rapidly growing domestic pigs can reach $96 \mathrm{~kg}$ live weight in 125 days. We can denote these figures genetic potential for rapid growth. Equation (9.1) is attributable to genetic potential phenotype for growth. It is reasonable quantitatively identify it with parameter $\mathrm{K}_{\mathrm{c}}$, namely 
$\mathrm{K}=2.50$ when $\mathrm{M}=96 \mathrm{~kg},\left.\mathrm{~K}\right|_{(\mathrm{K}=2.50) \wedge(\mathrm{M}=96)}=\mathrm{K}_{\mathrm{c}}$, and accordingly $\mathrm{t}_{(\mathrm{K}=2.50) \wedge(\mathrm{M}=96)}=\mathrm{t}_{\mathrm{c}}$. Analytical formulation of the growth rate considering the phenotype, equation $(9.1)$, given by

$$
\frac{1}{m_{o}} \cdot \frac{\Delta M}{\Delta t_{c}}=\frac{K_{c}}{t_{c}} \cdot \frac{3 K_{c} \cdot\left(2 K_{c}+1\right)-1}{\left(K_{c}+1\right)\left(2 K_{c}-1\right)}
$$

Numerically, equation $(9.2)$ gives growth rate $1.885 \mathrm{~kg}$ a day in the weight range between $30 \mathrm{~kg}$ and $96 \mathrm{~kg}$ live weight. Genetic potential for growth rate, equation (9.2) describes a distinct growth dynamic. Below, only one aspect, namely a very high momentane growth rate at $\mathrm{m}_{\mathrm{o}}$ is shown. I can remind the interested reader how to calculate momentane growth rate considering equation (9.2). One should notice that $\lim _{t_{c} \rightarrow t_{o}}\left(\frac{\Delta M}{\Delta t_{c}}\right)=\frac{d M}{d t}$. As a result, at the point $\left(\mathrm{m}_{0}=30\right) \wedge\left(\mathrm{t}_{\mathrm{c}}=\mathrm{t}_{0}=90\right)$, momentane growth rate considering equation (9.2) is $1.333 \mathrm{~kg}$ a day, considerably higher compared to other phenotypes. For example, equation (8) at the point $\left(\mathrm{m}_{0}=30\right) \wedge\left(\mathrm{t}_{\mathrm{c}}=\mathrm{t}_{\mathrm{o}}=90\right)$ gives growth rate $0.666 \mathrm{~kg}$ a day.

In the next stage, we consider systems (6) and (7). Taking into consideration feed conversion coefficient, $\mathrm{Z}$ the following growth rate equations were derived.

$$
\begin{gathered}
\frac{1}{m_{o}} \cdot \frac{\Delta M}{\Delta t}=\frac{1}{t} \cdot\left[\frac{2 K(Z-1)+Z}{2(Z-K)}\right], m_{o} \leq M \leq 96 . \mathrm{Z}>K . \\
\frac{1}{m_{o}} \cdot \frac{\Delta M}{\Delta t}=\frac{1}{t} \cdot\left(\frac{Z K}{Z-K}\right), m_{o} \leq M \leq 96 . \mathrm{Z}>K . \\
\frac{\Delta M}{\Delta t}=\frac{1}{t} \cdot\left[\frac{M Z K}{(2 K-1)(Z-K)}\right], m_{o} \leq M \leq 96 . \mathrm{Z}>K .
\end{gathered}
$$

Equations (10), (11) and (12) describe rapid growth. One can see that growth rate maximum is expected under condition $\mathrm{Z}=\mathrm{K}$. As minimum $\mathrm{K}=\mathrm{K}_{0}=1$, it follows from the heuristic notions that greatest growth rate is expected under condition $Z=1$. At this point $(Z=1)$, global maximum of growth rate has evidently been attained. However, under general model conditions the dynamic of feed conversion coefficient is highly nonlinear and as yet unclear.

We can add some more equations of rapid growth useful in practical applications.

$$
\begin{gathered}
\frac{1}{m_{o}} \cdot \frac{\Delta M}{\Delta t}=\frac{2 K^{2} t_{o}}{t^{2}}, m_{o} \leq M \leq 96 . \\
\frac{1}{m_{o}} \cdot \frac{\Delta M}{\Delta t}=\frac{2 K Z}{(Z-2 K) t+Z K t_{o}}, Z>K . m_{o} \leq M \leq 96 . \\
\frac{\Delta M}{\Delta t}=\frac{2 M K^{2} t_{o}}{(2 K-1) t^{2}}, m_{o} \leq M \leq 96 .
\end{gathered}
$$

3.1.2 Growth up to Maximum Weight

From system (4) one can derive the following equations.

$$
\begin{gathered}
\frac{1}{\mathrm{~m}_{\mathrm{o}}} \cdot \frac{\Delta \mathrm{M}}{\Delta \mathrm{t}}=\frac{\mathrm{K}}{\mathrm{t}} \cdot \frac{\mathrm{Z}(2 \mathrm{~K}+1)-2 \mathrm{~K}}{\mathrm{Z}(\mathrm{K}+1)-2 \mathrm{~K}}, \mathrm{~m}_{\mathrm{o}} \leq \mathrm{M} \leq \mathrm{M}_{\mathrm{x}}, \\
\frac{1}{m_{o}} \cdot \frac{\Delta M}{\Delta t}=\frac{1}{t} \cdot \frac{m_{o}[Z(2 K+1)-2 K]}{m_{o}[Z(2 K+1)-2 K]-M Z}, m_{o} \leq M \leq M_{x} .
\end{gathered}
$$

Considering equation (13) or (14), one can see that there is no growth if $Z(2 K+1)-2 K=0$. It means the condition under which animals do not growth at $m_{0}$ is $Z_{0}=2 / 3$. This is unexpected result, attributable to the nonlinearity of the growth process. Formal, linear logic says that $Z$ may not be less than 1 . In the range $Z>2 / 3$ growth in domestic pigs is observed. Before the next section some analyses is needed.

Data analyses acquired from pig-breeding farms where mature boars were kept proved that typical maximum individual weight was $M=M_{x}=600 \mathrm{~kg}$. Expression (15) converges as $M \rightarrow M_{x}$, and the following limit holds: 


$$
\lim _{M \rightarrow M_{x}}\left(\frac{2 K-1}{K+1}\right)=\sqrt{3} \text {, where }\left.\left(K \rightarrow K_{x}\right)\right|_{M \rightarrow M_{x}} .
$$

It follows from the limit that under condition $K=K_{x}, K_{x}=5+3 \sqrt{3}=10,19615$. In the next step, we calculate $Z_{\mathrm{x}},\left.Z\right|_{(M=M \mathrm{x}) \wedge(K=K \mathrm{x})}=Z_{\mathrm{x}}$. Considering system (4), the following equation was obtained.

$$
\frac{1}{m_{o}} \cdot \frac{\Delta M}{\Delta K}=\frac{2 K-1}{K}-\frac{2}{Z}
$$

Substituting $K_{x}$ and $M_{x}$ into (16) we have $Z_{x}=62.51$. The results above suggest the following facts: at $M_{x}=600 \mathrm{~kg}$, $\mathrm{K}_{\mathrm{x}}=10.19615$ and $\mathrm{Z}_{\mathrm{x}}=62.51$.

When maximum individual weight, $M_{x}$ has been reached growth stops. This means that at the point $\left(M_{x}, K_{x}, Z_{x}, t_{x}\right)$, feed conversion, $Z$ becomes infinity, $Z=\infty$. With this mathematical abstraction $(Z \rightarrow \infty)$ large, indefinable quantity of feed consumed at the maximum weight point was modelled; this follows from the reasoning that at maximum liveweight point, not any quantity of consumed feed can increase animals weight. This is also a bifurcation point. At this point, two new growth trajectories set to develop. In this paper, only one trajectory $\left(K \rightarrow K_{x} \rightarrow K_{1} \rightarrow K_{2}\right)$ is analysed. There is a tiny, indefinable border between $Z_{x}=62.51$ and $Z_{x}=\infty,\left.\left(t>t_{x}\right)\right|_{(M=M x)} \rightarrow Z=\infty$. The following analyses explains this aspect. Considering (16) and taking the limit $Z \rightarrow \infty$, we have

$$
\lim _{\mathrm{Z} \rightarrow \infty}\left(\frac{1}{m_{o}} \cdot \frac{\Delta M}{\Delta K}\right)=\frac{2 K+1}{K}, \text { where }\left.\left(K \rightarrow K_{1}\right)\right|_{Z \rightarrow \infty} .
$$

It follows from (17) that $\mathrm{K}_{1}=\left.\mathrm{K}\right|_{(M=M \mathrm{x}) \wedge(Z=\infty)}=10.04975$. If constants $\mathrm{M}_{\mathrm{x}}, \mathrm{K}_{\mathrm{x}}, \mathrm{Z}_{\mathrm{x}}$ and $\mathrm{t}_{\mathrm{x}}$ describe animals growth when individual maximum weight is reached, than constants $K_{1}$ and $t_{1}$ relate to $M_{x}$ under condition $Z=\infty$. In other words, in the growth stage $\left(\mathrm{K}_{\mathrm{x}} \rightarrow \mathrm{K}_{1}\right), \mathrm{M}_{\mathrm{x}}$ remains constant, while other variables change.

\subsection{Feed Conversion Coefficient}

Dynamic of feed conversion coefficient was not analysed in theoretical studies. However, feed conversion is relevant variable both for theoretical and practical applications. In this section, feed conversion coefficient, $Z$ has been analysed in weight range between $m_{o}$ and $M_{x}$. General dynamic of $Z$ is given by equations (13) and (14). Some more details are added in this section. $\left.Z\right|_{M=M_{x}}=Z_{x}$. Numerically, $Z_{\mathrm{x}}=62.51$. Analytical expression given by

$$
Z_{x}=\frac{2 K_{x}\left(K_{x}-1\right)}{3}
$$

For practical applications, especially in pig-breeding farms it is essential to know an average feed conversion coefficient of mature boars. We can offer an analytical formula to calculate average $\mathrm{Z}$ under condition that the boars have reached their maximum individual weight. Average $Z$ in the weight range from $m_{0}$ to $M_{x}$ we denote $\bar{Z}$. $\bar{Z}$ was found as integral average by considering $Z_{\mathrm{x}}$, equation (18). Considering $Z_{\mathrm{x}}$ and $\mathrm{K}_{\mathrm{x}}$ as variables we have

$$
\bar{z}=\frac{1}{K_{x}} \cdot \int Z_{x} d K_{x}=\frac{K_{x}}{3}\left(\frac{2 K_{x}}{3}-1\right)
$$

Numerically, $\bar{Z}=19.70$ for a boar $600 \mathrm{~kg}$ in weight. It follows from the model, that a boar $600 \mathrm{~kg}$ in weight, during its ontogeny consumed feed on average $5.0 \mathrm{~kg}$ a day. As a result, we can calculate an average daily gain for a boar that has reached $\mathrm{M}_{\mathrm{x}}$. It is $5.0 / 19.70=0.253 \mathrm{~kg}$ a day, numerically comparable with equations (13) and (21). Formula (19) and the method are applicable only to animals that reached their individual maximum weight. If it is not the case, the picture is significantly different. In this section we model an animal that cannot reach its individual maximum weight. As a model of such animal we consider the pig, which live weight was modelled as integral function of $\mathrm{M}_{\mathrm{x}}$, we denote it $\mathrm{M}_{a}$, given by

$$
\frac{M_{a}}{m_{o}}=2 K_{a}-\frac{4 K_{a}}{K_{x}^{2}}
$$

Numerically, $\mathrm{M}_{a}$ is $410.874 \mathrm{~kg}$. Formula (24) helps calculate other relevant parameters. So, $\left.\mathrm{K}\right|_{(M=M a)}=\mathrm{K}_{a}=6.9822$, and $\left.Z\right|_{(M=M a)}=Z_{a}=95.38$. Average $Z$, in this case we denote it $Z_{a a}$, numerically is 30.06 . One can see that an animal 
that cannot reach its individual maximum weight has completely different growth performance. However, if compare age at which the two considered animals reach $\mathrm{M}_{a}$ and $\mathrm{M}_{\mathrm{x}}$, respectively than we find that the age is equal, $\mathrm{t}_{\mathrm{K}=\mathrm{K} a}=\mathrm{t}_{a}=\mathrm{t}_{\mathrm{x}}=6.40$ years. Whereas life span of the compared modelled animals differ considerably. This suggests that in this case we study either diverse physiological conditions or a different phenotype. $Z_{x}$, the feed conversion coefficient found when an animal has reached its maximum individual weight can, in some weight range, be linearly extrapolated to other boars that reached their individual maximum weight. For example, in this manner calculated feed conversion coefficient for $500 \mathrm{~kg}$ heavy boar is 52.09 . This calculation is inappropriate for boars heavier than $600 \mathrm{~kg}$. Under the model conditions, animals heavier than $600 \mathrm{~kg}$ enter a different growth trajectory that is not analysed in this paper.

\subsection{Life Span}

At first, we shell find age of animals that reached their individual maximum weight, $\mathrm{M}_{\mathrm{x}}$. To do this we need result that follows from (15) and (1). Under condition $\left(\mathrm{M}=\mathrm{M}_{\mathrm{x}}\right) \wedge\left(\mathrm{K}=\mathrm{K}_{\mathrm{x}}\right)$, we have $\mathrm{t}_{\mathrm{x}}=6.40$ years. In formal notation $\left.t_{x}\right|_{\left(M=M_{x}\right) \wedge\left(K=K_{x}\right)}=6.40$ years. Analytical formulation given by

$$
\left\{\begin{array}{l}
\frac{M_{x}}{m_{o}}=2 K_{x}-\frac{4}{K_{x}} \\
\frac{t_{x}}{t_{o}}=\frac{K_{x}^{2}}{4}
\end{array}\right.
$$

From (20) and (18) follows growth rate, given by

$$
\frac{1}{m_{o}} \cdot \frac{\Delta M_{x}}{\Delta t_{x}}=\frac{3 K_{x}}{t_{x}} \cdot \frac{2 K_{x}\left(Z_{x}-1\right)+Z_{x}}{\left(3 Z_{x}-2 K_{x}\right)\left(K_{x}+2\right)}
$$

Taking the limit $\left(Z_{x} \rightarrow \infty\right)$ in (21) we get growth rate for an animal immediately after bifurcation of the growth trajectory.

$$
\lim _{Z_{x} \rightarrow \infty}\left[\frac{1}{m_{o}} \cdot \frac{\Delta M_{x}}{\Delta t_{x}}\right]=\frac{1}{t_{x}} \cdot \frac{K_{x}\left(2 K_{x}+1\right)}{K_{x}+2}
$$

In the next stage, we can find obtainable life span. For this task to complete, we use result that follows from (17) and (1). Under condition $\left(\mathrm{M}=\mathrm{M}_{\mathrm{x}}\right) \wedge\left(\mathrm{K}=\mathrm{K}_{1}\right), \mathrm{t}_{1}=24.90$ years. In formal notation $\left.t_{1}\right|_{\left(M=M_{x}\right) \wedge\left(K=K_{1}\right)}=24.90$ years. One should notice that in this case $\mathrm{Z}=\infty$. Analytical formulation given by

$$
\left\{\begin{array}{l}
\frac{M_{x}}{m_{o}}=2 K_{1}-\frac{1}{K_{1}} \\
\frac{t_{1}}{t_{o}}=K_{1}^{2}
\end{array}\right.
$$

From (22) follows growth rate during obtainable life span, we have

$$
\frac{1}{m_{o}} \cdot \frac{\Delta M_{x}}{\Delta t_{1}}=\frac{1}{t_{1}} \cdot \frac{K_{1}\left(2 K_{1}+1\right)}{K_{1}+1}
$$

I can remind the interested reader that under the model conditions, $\mathrm{M}_{\mathrm{x}}$ maximum individual weight remains constant in the time span between $t_{x}$ and $t_{2}$. Species maximum theoretical longevity $t_{2}$, follows from the mathematical abstraction applying passion to the limit $(\mathrm{t} \rightarrow \infty)$ procedure. It should be considered as the abstraction that sets time to be indefinitely long thus modelling the longest possible life span.

Considering $\mathrm{M}_{\mathrm{x}}$ and $\mathrm{K}_{\mathrm{x}}$ variables, I start analyses by writing well-known formula:

$$
M_{x} \cdot K_{x}=\int M_{x} d K_{x}+\int K_{x} d M_{x}
$$

In the next step, we shall find the limit of (1), given by

$$
\lim _{t \rightarrow \infty} K=\frac{M}{2 m_{o}} .
$$

It follows from the limit that $K_{2}=\left.K\right|_{t \rightarrow \infty}$ and

$$
d K=\frac{d M}{2 m_{o}}
$$


Substituting (25) in (24) we have

$$
K_{2}=\frac{M_{x}}{2 m_{o}}+\frac{m_{o}}{2 M_{x}}
$$

Numerically, $K_{2}=10.0250$. Under condition $\left(M=M_{x}\right) \wedge\left(K=K_{2}\right), t_{2}=49.31$ years. This is the species maximum theoretical longevity, $\mathrm{t}_{2}$. Analytical formulation given by

$$
\left\{\begin{array}{l}
\frac{M_{x}}{m_{o}}=K_{2}+\sqrt{K_{2}^{2}-1} \\
\frac{t_{2}}{t_{o}}=2 K_{2}^{2}-1
\end{array}\right.
$$

From (27) follows growth rate during maximum life span, given by

$$
\frac{1}{m_{O}} \cdot \frac{\Delta M_{x}}{\Delta t_{2}}=\frac{1}{t_{2}} \cdot\left[\frac{2 K_{2}^{2}-1}{2\left(K_{2}+1\right)}+\frac{2 K_{2}^{2}-1}{2 \cdot \sqrt{K_{2}^{2}-1}}\right], K_{2}>1 .
$$

One more fact is obtainable from (26). I re-write (26) considering $\mathrm{M}_{\mathrm{x}}$ variable, and $\mathrm{K}_{2}$ constant, given by

$$
\left(\frac{M_{x}}{m_{o}}\right)^{2}-2 K_{2} \frac{M_{x}}{m_{o}}+1=0
$$

Solution to the equation (29) is simple. One root is obvious, $M=M_{x}=600 \mathrm{~kg}$. Second root is more informative, $\mathrm{M}=\mathrm{m}_{\mathrm{b}}=0.05 \mathrm{~m}_{\mathrm{o}}=1.50 \mathrm{~kg} .1,5 \mathrm{~kg}$ is a birth weight of a healthy, vital piglet. This suggests that equation (26) comprises information about animal weight at birth, individual maximum weight, and maximum longevity.

The animals that cannot reach their maximum individual weight have significantly shorter life span. Such animals had been modelled considering weight $\mathrm{M}_{a}$. At this weight, animals stop growing not reaching maximum weight. It was shown that $\mathrm{t}_{a}=\mathrm{t}_{\mathrm{x}}$; however obtainable life span for these animals is 12.02 years, and maximum theoretical longevity is 23.79 years, pointing to a considerably shorter life span. This suggests that most pigs live less than 25 years.

\section{Discussions}

Since neither growth hormone nor insulin-like growth factor-1 enter the model as variable, a feasible link between the mentioned physiological factors and a feed conversion coefficient follows from the observation that many of the genetic pathways that control developmental growth in higher organisms - insulin, TOR signalling, and nuclear receptors-are also essential metabolic regulators (Tennessen \& Thummel, 2011).

\subsection{Ontogenetic Growth}

Modelling of biological systems, which is based on experimental data and aims to produce provable predictions, is decades long formulated task. This study is a model that clarifies some aspects underlying the growth and longevity in pigs. The model's results are experimentally testable; predictions derived from the model are consistent with empirical data, and field observations. The model entails the following three, in experiments found functional relations between analysed traits, formulated in equations (1), (2), and (4). System (4) describes functional relations between variables in the form that follows from experimental data analyses. Neither assumptions nor theoretical notions about growth were explicitly integrated in the model formulation. The results follow from the mathematical analyses of system (4), and its biological interpretation. It follows from the model, see equation (29), that a healthy piglet at birth is $1.5 \mathrm{~kg}$ in weight, and a mature 6.40 years old boar is $600 \mathrm{~kg}$ in weight. There is a considerable difference in the growth performance between animals that can reach their maximum weight and those that cannot. This suggests that animals ability reach their maximum individual weight is essential physiological trait that influences both growth performance and longevity. Under the model conditions, two sets of variables describe ontogenetic growth dynamic, namely $\left(\mathrm{K} \rightarrow \mathrm{K}_{\mathrm{x}} \rightarrow \mathrm{K}_{1} \rightarrow \mathrm{K}_{2}\right)$ and $\left(\mathrm{t}_{0} \rightarrow \mathrm{t} \rightarrow \mathrm{t}_{\mathrm{x}} \rightarrow \mathrm{t}_{1} \rightarrow \mathrm{t}_{2}\right)$. Both sets are functionally related to the set $\left(Z_{o} \rightarrow Z_{x} \rightarrow Z_{\infty}\right)$. The model suggests that growth and longevity are related traits.

\subsection{Longevity}

Longevity is not a variable in the model; it emerges as a result of growth dynamic analyses. This implies the relation between ontogenetic growth and life span partly demonstrated by the model. Moreover, the model suggests that these traits are interrelated. Our findings support the opinion that growth causes aging (Gems \& 
Partridge, 2013). In two cases, passion to the limit procedure was efficient in finding corresponding age. In case of maximum theoretical longevity, it was limit $(t \rightarrow \infty)$, and in case of obtainable life span, it was limit $(\mathrm{Z} \rightarrow \infty)$. Methods typically used in mathematical physics proved effective in this biological study as well.

It follows from the model that a mature boar reaches its maximum individual weight $600 \mathrm{~kg}$ in 6.40 years. The boar that had reached its maximum individual weight can reach its obtainable life span in 24.90 years. And, these animals can potentially live until aged 49.31 years, which is the species theoretical maximum longevity. We can denote these numbers genetic potential for life span in the pig.

\subsection{Feed Conversion}

In growing domestic pigs, feed conversion coefficient $Z$, varies from $Z_{0}=2 / 3$ up to theoretical abstraction - infinity. For those who accepted a heuristic perception of feed conversion coefficient this result is unexpected. However, linear logic cannot comprehend nonlinear processes. Moreover, feed conversion is so complicated trait that at this stage it is impossible outline its dynamic in detail.

\section{Conclusions}

- In domestic pigs, individual maximum weight is an essential physiological trait. Only animals that had reached their individual maximum weight have potential for reaching obtainable life span, species maximum weight, and subsequently species maximum longevity.

- Essential precondition of reaching obtainable life span is ability maintain individual maximum weight constant for years after maximum weight was reached.

- In terms of genetic potential, it is possible formulate the following limits. Growing domestic pigs perform genetic potential for growth with ability reach $96 \mathrm{~kg}$ live weight in 125 days, with average growth rate $1.885 \mathrm{~kg}$ a day between $30 \mathrm{~kg}$ and $96 \mathrm{~kg}$ live weight.

In the pig, obtainable life span is 24.90 years, and theoretical maximum longevity is 49.31 years implying that life span is genetically determined and related to growth.

- The feed conversion coefficient is the variable that provides a biological link between the growth processes at organism level and the biological functions at the lower physiological levels. In growing domestic pigs, feed conversion coefficient changes from $2 / 3$ up to theoretical abstraction - infinity.

- In the pig, cessation of growth rate in animals ontogeny is associated with dynamic of feed conversion coefficient. Ontogenetic growth stops when feed conversion coefficient grows into infinity.

- In the pig, life span is a function of growth rate and feed conversion coefficient.

\section{Conflict of interests}

The authors declare that there is no conflict of interests regarding the publication of this paper.

\section{References}

Agutter, P. S., \& Wheatley, D. N. (2004). Metabolic scaling: Consensus or controversy? Theoretical Biology and Medical Modelling, 1, 13.

Aihara, K., \& Suzuki, H. (2010). Theory of hybrid dynamical systems and its applications to biological and medical systems. Phil. Trans. R. Soc. A., 368, 4893-4914.

Barzilai, N., Huffman, D. M., Muzumdar, R. H., \& Bartke, A. (2012). The Critical Role of Metabolic Pathways in Aging. Diabetes, 61, 1315-1322.

Boukal, D. S., Dieckmann, U., Enberg, K., Heino, M., \& Jørgensen, C. (2014). Journal of Theoretical Biology, 359, 199-207.

Carter, C. S., Ramsey, M. M., Ingram, R. L., Cashion, A. B., ..., \& Sonntag, W. E. (2002). Models of Growth Hormone and IGF-1 Deficiency: Applications to Studies of Aging Processes and Life-Span Determination. Journal of Gerontology, 57A(5), B177-B188.

Di Bernardo, M., \& Hogan, S. J. (2010). Discontinuity-induced bifurcations of piecewise smooth dynamical systems. Phil. Trans. R. Soc. A., 368, 4915-4935.

Gems, D., \& Partridge, L. (2013). Genetics of Longevity in Model Organisms: Debates and Paradigm Shifts. The Annual Review of Physiology, 75, 621-44.

Heemels, P. M. H., De Schutter, B., Lunze, J., \& Lazar, M. (2010). Stability analysis and controller synthesis for hybrid dynamical systems. Phil. Trans. R. Soc. A., 368, 4937-4960. 
Lui, J. C., \& Baron, J. (2011). Mechanisms Limiting Body Growth in Mammals. Endocrine Reviews, 32, 422-440.

Mackay, T. F. C. (2001). The Genetic Architecture of Quantitative Traits. Annu. Rev. Genet, 35, 303-339.

Mitchell, S. J., Scheibye-Knudsen, M., Longo, D. L., \& de Cabo, R. (2015). Animal Models of Aging Research: Implications for Human Aging and Age-Related Diseases. The Annual Review of Animal Biosciences, 3, 283-303.

Nijhout, H. F. (2003). The control of growth. Development, 130, 5863-5867.

Nijhout, H. F. (2015). Big or fast: Two strategies in the developmental control of body size. BMC Biology, 13, 57.

Ostan, R., Monti, D., Gueresi, P., Bussolotto, M., ..., \& Baggio, G. (2016). Gender, aging and longevity in humans: An update of an intriguing/neglected scenario paving the way to a gender-specific medicine. Clinical Science, 130, 1711-1725.

Speakman, J. R. (2005). Body size, energy metabolism and life span. The Journal of Experimental Biology, 208, 1717-1730.

Suki, B., \& Frey, U. (2017). A time-varying biased random walk approach to human growth. Scientific Reports, 7, 7805.

Tennessen, J. M., \& Thummel, C. S. (2011). Coordinating Growth and Maturation - Insights from Drosophila. Current Biology, 21, R750-R757.

Vollmer, J., Casares, F., \& Iber, D. (2017). Growth and size control during development. Open Biol., 7, 170190.

Walters, E. M., Wolf, E., Whyte, J. J., Mao, J., ..., \& Prather, R. S. (2012). Completion of the swine genome will simplify the production of swine as a large animal biomedical model. BMC Medical Genomics, 5, 55.

Wu, R., Ma, C. X., Zhao, W., \& Casella, G. (2003). Functional mapping for quantitative trait loci governing growth rates: A parametric model. Physiol Genomics, 14, 241-249.

\section{Copyrights}

Copyright for this article is retained by the author(s), with first publication rights granted to the journal.

This is an open-access article distributed under the terms and conditions of the Creative Commons Attribution license (http://creativecommons.org/licenses/by/4.0/). 